\title{
THE COMPARISON OF SOME HYPERTOPOLOGIES
}

BY

\section{G. APREUTESEI}

\begin{abstract}
If $(X, d)$ is a metric space and $\mathcal{C l}(X)$ is the family of closed subsets of $X$, we search the "largest" family $\mathcal{A} \subset \mathcal{C l}(X)$ such that Vietoris topology $\tau_{V}$ and locally finite topology $\tau_{l f}$ coincide on $\mathcal{A}$. We also compare the convergences in Vietoris and bounded-Vietoris sense and in other hyperconvergences.

Mathematics Subject Classification 2000: 54B20.

Key words: hypertopology, hyperconvergence, locally finite topology, Vietoris topo$\log y$.
\end{abstract}

\section{Introduction}

In this paper we compare the locally finite and Vietoris topology on some classes of nonempty closed subset of a metric space. We find the "better" class with this property. Then we obtain another results in the problem of comparison of hypertopologies.

So consider $(X, d)$ a metric space. Denote by $\mathcal{P}(X)$ the family of all nonempty subsets of $X$. If $\mathcal{A} \subset \mathcal{P}(X)$, we define some topologies on $\mathcal{A}$ which extend the initial topology (see, for e.g., [3], [4], [7], [8], [12]). This means that, if we restrict these topologies to the family $\mathcal{S}(X)$ of singleton subsets of $X$, the topology of induced subspace agrees with the initial topology on $X$. These topologies are called hypertopologies or hyperspacial topologies. In this category one includes some well-known topologies, like Hausdorff topology $\tau_{H}$, Attouch-Wets topology $\tau_{A W}$, Vietoris and boundedVietoris topology ( $\tau_{v}$ and $\tau_{b V}$ respectively), proximal and bounded-proximal topology $\left(\tau_{P}\right.$ and $\tau_{b P}$ respectively), locally finite topology $\tau_{l f}$ and Wijsman topology $\tau_{W}$. 
On the family of the closed nonempty subsets $\mathcal{C l}(X)$ of $X$, there exist some relations between these hypertoplogies, as synthesized by SONNTAG and Z $\breve{A L I N E S C U}$ in [10] and [11]. We select below and adjust a fragment from the schema of this papers:

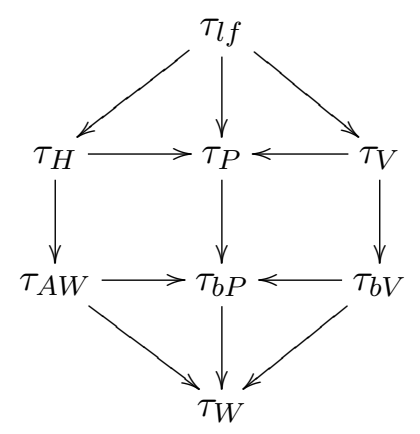

The relations described in schema above are generally strict. So many efforts have been done in order to find some conditions on the space $(X, d)$ which can assure the coincidence of these hypertopologies on $\mathcal{C l}(X)$. We give some of these results (which are specified in [5], [6] or [9]):

$\tau_{V}=\tau_{H} \Longleftrightarrow X$ is compact; $\tau_{V}=\tau_{P} \Longleftrightarrow(X, d)$ is UC space $([6]$, Lemma 5.2); $\tau_{V}=\tau_{l f} \Longleftrightarrow X$ is compact ([5], p.169); $\tau_{H}=\tau_{W} \Longleftrightarrow(X, d)$ is a totally bounded space ([6], Corollary 5.7); $\tau_{H}=\tau_{P} \Longleftrightarrow(X, d)$ is a totally bounded space ([6], Lemma 5.1); $\tau_{H}=\tau_{l f} \Longleftrightarrow(X, d)$ is UC space ([5], Theorem 2.2); $\tau_{P}=\tau_{W} \Longleftrightarrow(X, d)$ is a totally bounded space ([6], Theorem 5.5); $\tau_{P}=\tau_{l f} \Longleftrightarrow X$ is compact ([6], Section 5); $\tau_{W}=\tau_{l f} \Longleftrightarrow X$ is compact ([6], Corollary 5.8); $\tau_{V}=\tau_{b V} \Longleftrightarrow(X, d)$ is $d$-bounded ([9], Proposition 3.2); $\tau_{H}=\tau_{b V} \Longleftrightarrow X$ is compact ([9], Proposition 3.5); $\tau_{b V}=$ $\tau_{b P} \Longleftrightarrow(X, d)$ is BUC space $([9]$, Section 3$)$.

Recall that $(X, d)$ is a $U C$ space if every continuous real function on $X$ is uniformly continuous.

We say that $(X, d)$ is a $B U C$ space if for any $A, B \in \mathcal{C l}(X)$ and $B$ a bounded set, $A \cap B=\emptyset$ implies $d(A, B)>0$.

These results give an answer to the following question: to search special classes of metric spaces $(X, d)$ which make two specified hypertopologies to coincide on the fixed family $\mathcal{A}=\mathcal{C l}(X)$.

Another problem, initiated in [2], is to specify some family $\mathcal{A} \subset \mathcal{C l}(X)$ (even the largest family) on which two precise hypertopologies coincide, when the space $(X, d)$ is fixed. In this paper we focus especially on locally finite and Vietoris topologies. 
In Section 2 we recall some notations, notions and results concerning topologies from schema above.

Section 3 is dedicated to the comparison between locally finite and Vietoris topologies on $\mathcal{A} \subset \mathcal{C l}(X)$. We find the largest family on which they coincide.

In Section 4 we obtain a sufficiently condition for coincidence between the Vietoris and bounded Vietoris convergences of nets and finally we give some new conclusions on comparison between the hypertopologies defined in Section 2.

\section{Notations and preliminaries}

We consider a metric space $(X, d)$. Define the following families of nonempty subsets of $X$ :

$$
\begin{aligned}
& \mathcal{P}(X)=\{A \subset X ; A \neq \emptyset\} ; \\
& \mathcal{C} l(X)=\{A \in \mathcal{P}(X) ; A \text { is a closed subset }\} \\
& \mathcal{K}(X)=\{A \in \mathcal{P}(X) ; A \text { is a compact subset }\} \\
& \mathcal{F}(X)=\{A \in \mathcal{P}(X) ; A \text { is a finite subset }\} \\
& \mathcal{S}(X)=\{A \in \mathcal{P}(X) ; A \text { is a singleton }\} ; \\
& \mathcal{B}(X)=\{A \in \mathcal{C} l(X) ; A \text { is a } d \text {-bounded subset }\} ; \\
& \mathcal{P} k(X)=\{A \in \mathcal{P}(X) ; A \text { is a } d \text {-totally bounded subset }\}
\end{aligned}
$$

We denote by $S(a, \varepsilon)=\{x \in X ; d(a, x)<\varepsilon\}$ with $a \in X, \varepsilon>0$ the ball of center $a$ and radius $\varepsilon$ and by $B(a, \varepsilon)=\{x \in X ; d(a, x) \leq \varepsilon\}$ with $a \in X$, $\varepsilon>0$ the closed ball of center $a$ and radius $\varepsilon$.

$S_{\varepsilon}(A)$ is the notation of $\varepsilon$-enlargement of $A: S_{\varepsilon}(A)=\{x \in X ; \exists a \in A$ such that $d(x, a)<\varepsilon\}$ with $A \subset X, \varepsilon>0$.

The topologies which we consider in this paper must be written like a supremum of two topologies, namely a lower topology $\tau^{-}$and an upper topology $\tau^{+}$.

The Hausdorff topology $\tau_{H}$ is defined on $\mathcal{A} \subset \mathcal{C l}(X)$ by $\tau_{H}=\tau_{H}^{-} \vee \tau_{H}^{+}$, where a basic neighbourhoods of a set $A_{0} \in \mathcal{A}$ is, respectively:

in $\tau_{H}^{-}$ and in $\tau_{H}^{+}$

$$
U_{-}\left(A_{0}, \varepsilon\right)=\left\{A \in \mathcal{A} ; A_{0} \subset S_{\varepsilon}(A)\right\}, \text { with } \varepsilon>0,
$$

$$
U_{+}\left(A_{0}, \varepsilon\right)=\left\{A \in \mathcal{A} ; A \subset S_{\varepsilon}\left(A_{0}\right)\right\} \text {, with } \varepsilon>0 .
$$

The Attouch-Wets topology $\tau_{A W}$ on $\mathcal{A} \subset \mathcal{C l}(X)$ is $\tau_{A W}=\tau_{A W}^{-} \vee$ $\tau_{A W}^{+}$, where a basic neighbourhoods of a set $A_{0} \in \mathcal{A}$ is given by: 
in $\tau_{A W}^{-}$

and in $\tau_{A W}^{+}$

$$
U_{-}\left(A_{0} ; x_{0}, \varepsilon\right)=\left\{A \in \mathcal{A} ; A_{0} \cap S\left(x_{0}, \frac{1}{\varepsilon}\right) \subset S_{\varepsilon}(A)\right\}
$$

$$
U_{+}\left(A_{0} ; x_{0}, \varepsilon\right)=\left\{A \in \mathcal{A} ; A \cap S\left(x_{0}, \frac{1}{\varepsilon}\right) \subset S_{\varepsilon}\left(A_{0}\right)\right\},
$$

where $\varepsilon>0$ and $x_{0}$ is fixed arbitrarily in $X$.

The Vietoris topology $\tau_{V}$ on $\mathcal{A} \subset \mathcal{C l}(X)$ is $\tau_{V}=\tau_{V}^{-} \vee \tau_{V}^{+}$; a subbase for $\tau_{V}^{-}$is given by all the sets $V^{-}=\{A \in \mathcal{A} ; A \cap V \neq \emptyset\}$, where $V$ is an open subset of $X$, and a subbase for $\tau_{V}^{+}$is given by all the sets $E^{+}=\{A \in$ $\mathcal{A} ; A \cap E=\emptyset$, where $E$ is a closed subset of $X$.

The bounded-Vietoris topology ([9]) $\tau_{b V}$ on $\mathcal{A} \subset \mathcal{C l}(X)$ is $\tau_{b V}=$ $\tau_{V}^{-} \vee \tau_{b V}^{+}$; a subbase for $\tau_{b V}^{+}$is given by all the sets $E^{+}=\{A \in \mathcal{A} ; A \cap E=\emptyset\}$, where $E$ is a closed and bounded subset of $X$.

The proximal topology $\tau_{P}$ on $\mathcal{A} \subset \mathcal{C l}(X)$ is $\tau_{P}=\tau_{V}^{-} \vee \tau_{P}^{+}$, where $\tau_{P}^{+}$ is generated by all the sets $E^{++}=\left\{A \in \mathcal{A} ; \exists \varepsilon>0\right.$ such that $\left.S_{\varepsilon}(A) \subset E\right\}$ with $E$ open in $X$.

In fact, $\tau_{P}^{+}=\tau_{H}^{+}$.

The bounded-proximal topology $\tau_{b P}$ on $\mathcal{A} \subset \mathcal{C l}(X)$ is $\tau_{b P}=\tau_{V}^{-} \vee$ $\tau_{A W}^{+}$.

The Wijsman topology $\tau_{W}$ on $\mathcal{A} \subset \mathcal{C l}(X)$ is $\tau_{W}=\tau_{V}^{-} \vee \tau_{W}^{+}$, where the basic neighbourhoods of $A_{0}$ in upper Wijsman topology are the sets

$$
\left\{A \in \mathcal{A} ; d\left(x_{i}, A_{0}\right)<d\left(x_{i}, A\right)+\varepsilon \text { for } i=\overline{1, n}\right\},
$$

with $\left\{x_{1}, x_{2}, \ldots, x_{n}\right\}$ a finite subset of $X$ and $\varepsilon>0$.

The locally finite topology $\tau_{l f}$ on $\mathcal{A} \subset \mathcal{C l}(X)$ is $\tau_{l f}=\tau_{l f}^{-} \vee \tau_{V}^{+}$, where $\tau_{l f}^{-}$is generated by all the sets $\mathcal{L}^{-}=\{A \in \mathcal{A} ; A \cap V \neq \emptyset$, for every $V \in \mathcal{L}\}$, for any locally finite family $\mathcal{L} \subset \mathcal{P}(X)$ of open subsets of $X$.

(A family of subsets $\mathcal{L} \subset \mathcal{P}(X)$ is called locally finite if for every $x \in$ $X$ there exists a neighbourhood $V(x)$ of $x$ such that $V(x)$ has nonempty intersection with a finite number of elements of $\mathcal{L}$.)

We note that Vietoris and locally finite topologies do not depend on the metric of the space (like Hausdorff, Attouch-Wets, proximal, boundedproximal, bounded-Vietoris and Wijsman topologies), but they depend on the topology of the space. 
3. The comparison between the locally finite and Vietoris

\section{topologies}

Now we search the family $\mathcal{A} \subset \mathcal{C l}(X)$ such that $\tau_{V}$ and $\tau_{l f}$ coincide on $\mathcal{A}$. The upper topology for $\tau_{V}$ and $\tau_{l f}$ is the same, so we are interested by the hypertopologies $\tau_{l f}^{-}$and $\tau_{V}^{-}$. First we give the following lemma:

Lemma 3.1. Let $(X, d)$ be an arbitrary metric space and $\mathcal{A} \subset \mathcal{C l}(X)$ be a class of sets such that for every locally finite family $\mathcal{L}$ of open subsets of $X$, the condition $\mathcal{L}^{-} \cap \mathcal{A} \neq \emptyset$ implies that $\mathcal{L}$ is finite. Then $\tau_{V}^{-}=\tau_{\text {lf }}^{-}$on $\mathcal{A}\left(\right.$ so $\tau_{V}=\tau_{\text {lf }}$ on $\left.\mathcal{A}\right)$.

Proof. We suppose that $\mathcal{L}$ is a locally finite family of open subsets of $X$ such that $\mathcal{L}^{-} \cap \mathcal{A} \neq \emptyset$. Let $V_{1}, V_{2}, \ldots, V_{n}$ be the open sets such that $\mathcal{L}=\left\{V_{1}, V_{2}, \ldots, V_{n}\right\}$. Then $\mathcal{L}^{-}=V_{1}^{-} \cap V_{2}^{-} \cap \ldots \cap V_{n}^{-}$is a finite intersection of open sets from the subbase of $\tau_{V}^{-}$, so $\mathcal{L}^{-}$is a $\tau_{V}^{-}$-open set. We deduce that $\tau_{l f}^{-} \subset \tau_{V}^{-}$, so $\tau_{l f} \subset \tau_{V}$. The reverse inclusion is valid on every class $\mathcal{A} \subset$ $\mathcal{C l}(X)$ (see the schema of section 1 ).

Remark 3.1. There exist some classes $\mathcal{A} \subset \mathcal{C l}(X)$ such that for every locally finite family $\mathcal{L}$ the condition $\mathcal{L}^{-} \cap \mathcal{A} \neq \emptyset$ implies that $\mathcal{L}$ is finite.

For example, in the case of the class $\mathcal{S}(X)$ we have: if $\mathcal{L}$ is an arbitrary locally finite family for which $\mathcal{L}^{-} \cap \mathcal{S}(X) \neq \emptyset$, then there exists $x_{0} \in X$ such that $\left\{x_{0}\right\} \in \mathcal{L}^{-}$. So $\left\{x_{0}\right\} \cap V \neq \emptyset$ for all $V \in \mathcal{L}$. But $\mathcal{L}$ is a locally finite family; hence there exists $U\left(x_{0}\right)$ a neighbourhood of $x_{0}$ which has a nonempty intersection with a finite number of elements of $\mathcal{L}$. Let they be $V_{1}, V_{2}, \ldots, V_{n}$. Then $U\left(x_{0}\right) \cap V \neq \emptyset$ for all $V \in \mathcal{L}$, so $\mathcal{L}$ contains only $V_{1}$, $V_{2}, \ldots, V_{n}$.

Another family $\mathcal{A} \subset \mathcal{C l}(X)$ which has the property from Lemma 3.1 is $\mathcal{A}=\mathcal{K}(X)$ :

Lemma 3.2. Let $(X, d)$ be a metric space. If $\mathcal{L}$ is a locally finite family of open subsets of $X$ and $\mathcal{L}^{-} \cap \mathcal{K}(X) \neq \emptyset$, then $\mathcal{L}$ contains a finite number of elements.

Proof. We suppose by contradiction that $\mathcal{L}^{-} \cap \mathcal{K}(X) \neq \emptyset$ and $\mathcal{L}$ has an infinite number of elements. Let $A$ be a compact subset of $X$ such that $A \in \mathcal{L}^{-}$. Then $A \cap V \neq \emptyset$ for all $V \in \mathcal{L}$. We choose $\left(V_{n}\right)_{n \in \mathbb{N}^{*}} \subset \mathcal{L}$ and let $x_{n} \in V_{n} \cap A, n \in \mathbb{N}^{*}$. (We denote by $\mathbb{N}^{*}$ the set of positive integer $\mathbb{N} \backslash\{0\}$.) 
Because $A$ is a compact set, hence a sequential compact set, there exists a subsequence $\left(x_{n_{k}}\right)_{k \in \mathbb{N}^{*}}$ and $x_{0} \in A$ with $x_{n_{k}} \rightarrow x_{0}$. For $x_{0}$ there exists $U\left(x_{0}\right)$ a neighbourhood which has a nonempty intersection with a finite number $p$ of elements of $\mathcal{L}$. Eventually by renumbering the sets of $\mathcal{L}$, let they be $V_{1}$, $V_{2}, \ldots, V_{p}$. Now we denote again the sequence $\left(V_{n_{k}}\right)_{k \in \mathbb{N}^{*}} \backslash\left\{V_{1}, V_{2}, \ldots, V_{p}\right\}$ by $\widetilde{V}_{n_{1}}, \widetilde{V}_{n_{2}}, \ldots, \widetilde{V}_{n_{k}}, \ldots$ and by $\widetilde{x}_{n_{k}}$ the elements of the sequence $\left(x_{n_{k}}\right)_{k \in \mathbb{N}^{*}}$ from $\widetilde{V}_{n_{k}} \cap A$. So $\widetilde{x}_{n_{k}} \rightarrow x_{0}$. For the neighbourhood $U\left(x_{0}\right)$ there exists $k_{0} \in \mathbb{N}^{*}$ such that for every $n_{k} \geq n_{k_{0}}$ we have $\widetilde{x}_{n_{k}} \in U\left(x_{0}\right)$. Hence $U\left(x_{0}\right)$ has a nonempty intersection with an infinite number of subsets of $\mathcal{L}$. This contradiction proves the lemma.

Theorem 3.3. Let $(X, d)$ be a metric space. On the class $\mathcal{K}(X)$ of compact subsets of $X$ we have $\tau_{V}^{-}=\tau_{l f}^{-}$or, equivalently, $\tau_{V}=\tau_{l f}$.

Proof. This is an immediate consequence of Lemma 3.1 and Lemma 3.2 .

Remark 3.2. If $(X, d)$ is a compact metric space, then $\mathcal{C l}(X)=\mathcal{K}(X)$ and hence $\tau_{V}=\tau_{l f}$ on $\mathcal{C l}(X)$. So we obtain from Theorem 3.3 the coincidence between $\tau_{V}$ and $\tau_{l f}$ on $\mathcal{C l}(X)$, a known result (see, for example, [5], p.169).

Now we intend to find the largest class $\mathcal{A} \subset \mathcal{C l}(X)$ on which $\tau_{V}^{-}=\tau_{l f}^{-}$. When we study the same problem for another pair of hypertopologies $\left(\tau_{H}^{-}\right.$ and $\tau_{A W}^{-}, \tau_{H}^{+}$and $\tau_{V}^{+}, \tau_{H}^{-}$and $\tau_{V}^{-}$etc.) we obtain (in [2]) the classes $\mathcal{B}(X)$, $\mathcal{P} k(X)$ and $\mathcal{K}(X)$. So one requires that the class $\mathcal{A}$ contains only bounded subsets. To give an answer to this question, we first prove the following lemma:

Lemma 3.4. Let $(X, d)$ be a metric space and $\mathcal{A} \subset \mathcal{C l}(X)$ be such that $\mathcal{A} \backslash \mathcal{B}(X) \neq \emptyset$. Then we can construct a locally finite family $\mathcal{L}$ of open unbounded subsets of $X$ such that $\mathcal{A} \backslash \mathcal{B}(X)=\mathcal{L}^{-}$.

Proof. Let $A_{0} \in \mathcal{A} \backslash \mathcal{B}(X)$. We fix $a \in X$. Since $A_{0}$ is unbounded subset of $X$, for every $n \in \mathbb{N}^{*}$ there exists $a_{n} \in A_{0}$ such that $d\left(a_{n}, a\right)>n$. So $a_{n} \in B(a, n)^{c}$, where $B(a, n)^{c}$ denotes the complement of the closed ball $B(a, n)$. The set $V_{n}=B(a, n)^{c}$ is open and unbounded in $X$ and $A_{0} \cap V_{n} \neq \emptyset$. Now we put $\mathcal{L}=\left(V_{n}\right)_{n \in \mathbb{N}^{*}}$ and we prove that $\mathcal{L}$ is a locally finite family:

Let $x \in X$ be an arbitrary point. Denote by $n_{0}=[d(a, x)]$ the integer with the property $n_{0} \leq d(a, x)<n_{0}+1$. Let $\varepsilon=n_{0}+1-d(a, x)$ be 
a positive number. Then $S(x, \varepsilon)$ is for $\mathcal{L}$ the desired neighbourhood of $x$ from the definition of locally finite family: if $y \in S(x, \varepsilon)$ then $d(a, y) \leq$ $\varepsilon+d(a, x)=n_{0}+1$, so $y \notin V_{n_{0}+1}$. Also $y \notin V_{k}$, where $k \geq n_{0}+1$. Hence $y$ is contained at most in $V_{1}, V_{2}, \ldots, V_{n_{0}}$. So the neighbourhood $S(x, \varepsilon)$ of $x$ intersects a finite number of elements of $\mathcal{L}$.

Now we can give the result:

Proposition 3.5. Let $(X, d)$ be a metric space and $\mathcal{A} \subset \mathcal{C l}(X)$ be a nonvoid class such that $\tau_{V}^{-}=\tau_{\text {lf }}^{-}$on $\mathcal{A}$. Then $\mathcal{A} \subset \mathcal{B}(X)$.

Proof. We suppose by contradiction that $\mathcal{A} \nsubseteq \mathcal{B}(X)$. Then $\mathcal{A} \backslash \mathcal{B}(X) \neq$ $\emptyset$. From the proof of Lemma 3.4 we have a locally finite family $\mathcal{L}=\left(V_{n}\right)_{n \in \mathbb{N}}$ of open and unbounded subsets of $X$, where $V_{n}=B(a, n)^{c}$ and $a$ is fixed in $X$. Observe that $\mathcal{L}^{-}$is open in $\left(\mathcal{A}, \tau_{l f}^{-}\right)$. Now if $A \in \mathcal{L}^{-}$, then $A \cap V_{n} \neq \emptyset$, for every $n \in \mathbb{N}^{*}$, so $A$ is unbounded and $\mathcal{L}^{-}$contains only unbounded closed subsets of $X$.

Since $\tau_{V}^{-}=\tau_{l f}^{-}$on $\mathcal{A}$, it follows that $\mathcal{L}^{-}$is $\tau_{V}^{-}$-open; we have $\mathcal{L}^{-}=$ $\bigcup_{t \in T} \mathcal{W}_{t}$, where $\mathcal{W}_{t}=\left(W_{1}^{t}\right) \cap\left(W_{2}^{t}\right) \cap \ldots \cap\left(W_{k}^{t}\right), k \in \mathbb{N}^{*}$ and $W_{1}^{t}, W_{2}^{t}, \ldots, W_{k}^{t}$ are open subsets of $X$. We choose $a_{1}^{t} \in W_{1}^{t}, \ldots, a_{k}^{t} \in W_{k}^{t}$. The set $A=\left\{a_{1}^{t}\right.$, $\left.\ldots, a_{k}^{t}\right\} \in \mathcal{B}(X)$ has the property that $A \cap W_{j}^{t} \neq \emptyset$ for every $j \in\{1,2, \ldots, k\}$. This condition implies that $A \in \mathcal{W}_{t}$, so $A \in \mathcal{L}^{-}$. This is a contradiction, because $\mathcal{L}^{-}$contains only unbounded closed subsets of $X$.

Remark 3.3. If there exists the largest family $\mathcal{A} \subset \mathcal{C l}(X)$ for which $\tau_{V}^{-}=\tau_{l f}^{-}$, then by Lemma 3.2 and Proposition 3.5, we have $\mathcal{K}(X) \subset$ $\mathcal{A} \subset \mathcal{B}(X)$. In fact $\mathcal{A} \varsubsetneqq \mathcal{B}(X)$. Indeed, assume by contrary that $\mathcal{A}=\mathcal{B}(X)$. Since $\tau_{V}$ and $\tau_{l f}$ are not dependent on the metric of the space (but they are depended on the topology of the space), replacing $d$ by $\frac{d}{1+d}$, it follows that the two topologies coincide on every metric space. This contradiction proves the above assertion.

Definition 3.1. A metric space $(X, d)$ is called boundedly compact if every closed and $d$-bounded subset of $X$ is compact.

Corollary 3.6. If $(X, d)$ is a boundedly compact space, then the largest class $\mathcal{A} \subset \mathcal{C l}(X)$ for which $\tau_{V}^{-}=\tau_{l f}^{-}\left(\right.$i.e. $\left.\tau_{V}=\tau_{l f}\right)$ on $\mathcal{A}$ is $\mathcal{K}(X)$.

In order to prove a similar result on an arbitrary metric space, we recall the following definition introduced in [2]: 
Definition 3.2. A family $\mathcal{A} \subset \mathcal{C l}(X)$ of parts of a metric space $X$ is called stable with respect to closed subsets if for any set $A \in \mathcal{A}$ and $B \subset A$, with $B \in \mathcal{C l}(X)$ it follows that $B \in \mathcal{A}$.

Remark 3.4. All the families $\mathcal{B}(X), \mathcal{P} k(X), \mathcal{K}(X), \mathcal{F}(X)$ are stable with respect to closed subsets.

Theorem 3.7. Let $(X, d)$ be a metric space and

$$
\begin{aligned}
& \mathcal{E}=\left\{\mathcal{A} \subset \mathcal{C l}(X) ; \mathcal{A} \neq \emptyset \text { and } \tau_{V}^{-}=\tau_{\text {lf }}^{-} \text {on } \mathcal{A},\right. \\
&\mathcal{A} \text { stable with respect to closed subsets }\} .
\end{aligned}
$$

Then $\mathcal{K}(X)$ is the largest element of $\mathcal{E}$.

Proof. From Theorem 3.3 it follows that $\mathcal{K}(X) \in \mathcal{E}$.

Now we show that $\mathcal{K}(X)$ is upper bound element for class $\mathcal{E}$.

We suppose by contradiction that $\mathcal{A} \varsubsetneqq \mathcal{K}(X)$ for some $\mathcal{A} \in \mathcal{E}$ and let $A \in$ $\mathcal{A} \backslash \mathcal{K}(X)$. So there exists a sequence $\left(a_{n}\right)_{n \in \mathbb{N}^{*}} \subset A$ such that $\left(a_{n}\right)_{n \in \mathbb{N}^{*}}$ does not have cluster points. We consider the subsets $A_{0}=\left\{a_{1}, a_{2}, \ldots, a_{k}, \ldots\right\}$ and $A_{k}=\left\{a_{1}, a_{2}, \ldots, a_{k}\right\}$. Since $A_{k}$ is finite, $A_{k}$ is closed. The set of cluster points of $A_{0}$ is $\emptyset$, so $A_{0}$ is closed too. Obviously, $A_{0}, A_{k} \subset A \in \mathcal{A}$ which is stable with respect to closed sets, hence $A_{0}, A_{k} \in \mathcal{A}$.

Now we observe that $A_{0} \in \tau_{V}^{-}-\lim _{k \rightarrow \infty} A_{k}$ : for any open set $V$ with $V \cap A_{0} \neq \emptyset$, there exists an $a_{k_{V}} \in V \cap A_{0}$. One deduces that for every $k \geq k_{V}$ we have $a_{k_{V}} \in V \cap A_{k}$.

We show that $A_{0} \notin \tau_{l f}-\lim _{k \rightarrow \infty} A_{k}$.

First we can construct a family $\mathcal{L}_{0}$ composed by all the open spheres $V_{k}=S\left(a_{k}, 1 / k\right)$; it comes out that $\mathcal{L}_{0}$ is a locally finite family. Indeed, we suppose that there exists an $a_{0} \in X$ such that for any $n \in \mathbb{N}^{*}, S\left(a_{0}, 1 / n\right)$ intersects an infinite number of $V_{k}$, say $V_{k_{p}}$. So there exists $b_{n}^{p} \in S\left(a_{0}, 1 / n\right) \cap$ $S\left(a_{k_{p}}, 1 / k_{p}\right)$. Then $d\left(a_{k_{p}}, a_{0}\right) \leq d\left(a_{k_{p}}, b_{n}^{p}\right)+d\left(b_{n}^{p}, a_{0}\right)<\left(1 / k_{p}\right)+(1 / n)$. Passing to the limit as $n \rightarrow \infty$, one derives that $d\left(a_{k_{p}}, a_{0}\right) \leq 1 / k_{p}$, hence $a_{k_{p}} \rightarrow a_{0}$. This is a contradiction, because $\left(a_{k}\right)_{k \in \mathbb{N}^{*}}$ has not cluster points.

It is obviously that $A_{0} \in \mathcal{L}_{0}^{-}: a_{k} \in A_{0} \cap V_{k}$ for every $k \in \mathbb{N}^{*}$.

Now we prove that for any $n_{0} \in \mathbb{N}^{*}$, there exists $n \geq n_{0}$ with $A_{n} \notin$ $\mathcal{L}_{0}$; more exactly if we fix $n_{0}$, we can find $k_{0}$ such that $\left\{a_{1}, a_{2}, \ldots a_{n_{0}}\right\} \cap$ $S\left(a_{k_{0}}, 1 / k_{0}\right)=\emptyset$. If we suppose by contradiction that for every $k \in \mathbb{N}^{*}$, $\left\{a_{1}, a_{2}, \ldots, a_{n_{0}}\right\} \cap S\left(a_{k}, 1 / k\right) \neq \emptyset$, there exists $j_{0} \in\left\{1, \ldots, n_{0}\right\}$ and a infinite number of terms $k_{p} \in \mathbb{N}^{*}$ such that $d\left(a_{k_{p}}, a_{j_{0}}\right)<1 / k_{p}$, so $a_{k_{p}} \rightarrow a_{j_{0}}$. The contradiction we arrived at, proves the theorem. 


\section{Some consequences}

In this section, we compare Vietoris and bounded Vietoris convergences on $\mathcal{A} \subset \mathcal{P}(X)$. In fact we want to find sufficient conditions on a class $\mathcal{A} \subset$ $\mathcal{P}(X)$, such that $\tau_{V}^{+}$-convergence and $\tau_{b V^{+}}^{+}$-convergence coincide. We recall that $\tau_{V}^{+}=\tau_{b V}^{+}$if and only if $(X, d)$ is $d$-bounded (see Proposition 3.2 from $[9])$. But we have not the coincidence of these topologies on $\mathcal{B}(X)$. For our study we first give the following definition.

Definition 4.1. A net $\left(A_{i}\right)_{i \in I} \in \mathcal{A} \subset \mathcal{P}(X)$ is said to be uniformly bounded net if there exists a closed ball $B(a, r) \subset X$ such that $A_{i} \subset B(a, r)$ for all $i \in I$, where $a \in X$ and $r>0$.

Remark 4.1. If $\left(A_{i}\right)_{i \in I} \in \mathcal{P}(X)$ is a uniformly bounded net, then $A_{i}$ is bounded for any $i \in I$.

Proposition 4.1. Let $(X, d)$ be a metric space and $A \in \mathcal{B}(X)$. If $\left(A_{i}\right)_{i \in I}$ is a uniformly bounded net $\tau_{b V}^{+}$-convergent to $A$, then $\left(A_{i}\right)_{i \in I}$ is also $\tau_{V}^{+}$ convergent to $A$ (and thus the $\tau_{b V}$-convergence of a uniformly bounded net with bounded limit coincides with $\tau_{V}$-convergence of them).

Proof. Let $B(a, r)$ be a closed ball such that $A, A_{i} \subset B(a, r)$ for any $i \in I$ and $G$ be a closed set of $X$ such that $A \cap G=\emptyset$. But $A \cap G=$ $(A \cap B(a, r)) \cap G=A \cap(B(a, r) \cap G)$. Since $A \in \tau_{b V^{-}}^{+} \lim A_{i}$, we use the closed and $d$-bounded set $E=B(a, r) \cap G$ in the definition of $\tau_{b V}^{+}$-neighbourhoods of $A$. Then there exists $i_{E} \in I$ such that $A_{i} \cap(B(a, r) \cap G)=\emptyset$ for every $i \geq i_{E}$. We put $i_{G}=i_{E}$ and we obtain that $A \in \tau_{V}^{+}-\lim A_{i}$.

Corollary 4.2. If $(X, d)$ is a metric space without isolated points and $\left(A_{i}\right)_{i \in I} \in \mathcal{K}(X)$ is a uniformly bounded net, then for a given $A \in \mathcal{K}(X)$, the following convergences are equivalent:

i) $A \in \tau_{b V}^{+}-\lim A_{i}$;

ii) $A \in \tau_{V}^{+}-\lim A_{i}$;

iii) $A \in \tau_{H}^{+}-\lim A_{i}$;

iv) $A \in \tau_{A W}^{+}-\lim A_{i}$. 
Proof. $i) \Longleftrightarrow i i$ ) is Proposition 4.1 (applied on the subclass $\mathcal{K}(X)$ ).

ii) $\Longleftrightarrow$ iii) follows from [2], Theorem 6.2: on $\mathcal{K}(X)$ we have $\tau_{V}^{+}=$ $\tau_{H}^{+}$when $(X, d)$ is a metric space without isolated points. (Therefore the uniformly bounded condition for the net $\left(A_{i}\right)_{i \in I}$ is not necessary.)

iii $) \Longleftrightarrow i v$ ) is immediately from [2], Proposition 4.1, (ii): if $\left(A_{i}\right)_{i \in I} \subset$ $\mathcal{C l}(X)$ is uniformly bounded net, the $\tau_{A W^{-}}^{+}$-convergence coincides with $\tau_{H^{-}}^{+}$ convergence on $\mathcal{C l}(X)$ (also on $\mathcal{K}(X)$ ).

Corollary 4.3. Let $(X, d)$ be a metric space and $A, A_{i} \in \mathcal{K}(X)$, for any $i \in I$. Then we have the equivalence for the following convergences:

i) $A \in \tau_{V}^{-}-\lim A_{i}$;

ii) $A \in \tau_{l f}^{-}-\lim A_{i}$;

iii) $A \in \tau_{A W}^{-}-\lim A_{i}$;

iv) $A \in \tau_{H}^{-}-\lim A_{i}$.

Proof. The assertion $i) \Leftrightarrow i i$ ) results from Theorem 3.3, for an arbitrary net.

The $\tau_{V}^{-}$-convergence coincides with the $\tau_{A W^{-}}^{-}$-convergence on the class $\mathcal{P} k(X) \supset \mathcal{K}(X)$. $\mathcal{K}(X)$.

The $\tau_{A W^{-}}^{-}$-convergence and the $\tau_{H}^{-}$-convergence are the same on $\mathcal{B}(X) \supset$

Corollary 4.4. If $A \in \mathcal{K}(X)$ and $\left(A_{i}\right)_{i \in I} \subset \mathcal{K}(X)$ is a uniformly bounded net from a metric space $(X, d)$ without isolated points, then the following assertions are equivalent:

i) $A=\tau_{b V}-\lim A_{i}$;

ii) $A=\tau_{V}-\lim A_{i}$;

iii) $A=\tau_{H}-\lim A_{i}$;

iv) $A=\tau_{A W}-\lim A_{i}$;

v) $A=\tau_{P}-\lim A_{i}$;

vi) $A=\tau_{b P}-\lim A_{i}$. 
(The proof follows from Corollaries 4.2 and 4.3.)

Corollary 4.5. On the class $\mathcal{K}(X)$ of compact subsets of a metric space $(X, d)$ without isolated points, the following relations between hyperconvergences are valid:

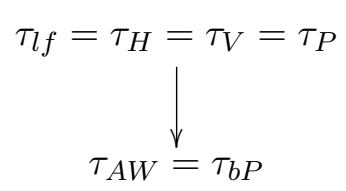

For the monotone sequences of subsets of $\mathcal{K}(X)$ all this convergences coincide with $\tau_{W}$.

Proof. The results are consequences of Theorem 3.3 above and of Corollary 5.5 and Theorem 6.2 from [2]. Also we use Theorem 3.1 from [1].

\section{REFERENCES}

1. Apreutesei, G. - Set convergence and the class of compact subsets, An. Ştiinţ. Univ. "Al. I. Cuza" Iaşi. Mat. (N.S.), 47 (2001), 263-276.

2. Apreutesei, G. - Families of subsets and the coincidence of hypertopologies, An. Ştiinţ. Univ. "Al. I. Cuza" Iaşi. Mat. (N.S.), 49 (2003), 3-18.

3. Аттоuch, H. - Variational Convergence for Functions and Operators, Applicable Mathematics Series, Pitman (Advanced Publishing Program), Boston, MA, 1984.

4. Beer, G.A.; Himmelberg, C.J.; Prikry, K.; Van Vleck, F.S. - The locally finite topology on $2^{X}$, Proc. Amer. Math. Soc., 101 (1987), 168-172.

5. Beer, G.; Lechicki, A.; Levi, S.; Naimpally, S. - Distance functionals and suprema of hyperspace topologies, Ann. Mat. Pura Appl., 162 (1992), 367-381.

6. Beer, G. - Topologies on Closed and Closed Convex Sets, Mathematics and its Applications, 268, Kluwer Academic Publishers Group, Dordrecht, 1993.

7. Castaing, C.; Valadier, M. - Convex analysis and measurable multifunctions, Lecture Notes in Mathematics, Vol. 580, Springer-Verlag, Berlin-New York, 1977.

8. Klein, E.; Thompson, A.C. - Theory of Correspondences. Including Applications to Mathematical Economics, Canadian Mathematical Society Series of Monographs and Advanced Texts, A Wiley-Interscience Publication, John Wiley \& Sons, Inc., New York, 1984. 
9. Lucchetti, R.; Pasquale, A. - The bounded Vietoris topology and applications, Ricerche Mat., 43 (1994), 61-78.

10. Sonntag, Y.; ZăLinescu, C. - Set convergences. An attempt of classification, Trans. Amer. Math. Soc., 340 (1993), 199-226.

11. Sonntag, Y.; ZăLinescu, C. - Set convergences: a survey and a classification. Set convergence in nonlinear analysis and optimization, Set-Valued Anal., 2 (1994), 339-356.

12. WiJsman, R.A. - Convergence of sequences of convex sets, cones and functions II, Trans. Amer. Math. Soc., 123 (1966), 32-45. 